\title{
Control of Statically Hoverable Multi-Rotor Aerial Vehicles and Application to Rotor-Failure Robustness for Hexarotors
}

\author{
Giulia Michieletto $^{1,2}$, Markus Ryll ${ }^{1}$ and Antonio Franchi ${ }^{1}$
}

\begin{abstract}
Standard hexarotors are often mistakenly considered 'by definition' fail-safe multi-rotor platforms because of the two additional propellers when compared to quadrotors. However this is not true, in fact, a standard hexarotor cannot statically hover with 'only' five propellers. In this paper we provide a set of new general algebraic conditions to ensure static hover for any multi-rotor platform with any number of generically oriented rotors. These are elegantly formulated as the full-rankness of the control moment input matrix, and the non-orthogonality between its null-space and the row space of the control force input matrix. Input saturations and safety margins are also taken into account with an additional condition on the null-space of control moment input matrix. A deep analysis on the hoverability properties is then carried out focusing on the propeller loss in a hexarotor platform. Leveraging our general results we explain why a standard hexarotor is not robust and how it can be made robust thanks to a particular tilt of the rotors. We finally propose a novel cascaded controller based on a preferential direction in the null-space of the control moment input matrix for the large class of statically hoverable multi-rotors, which goes far beyond standard platforms, and we apply this controller to the case of failed tilted hexarotor.
\end{abstract}

\section{INTRODUCTION}

Nowadays the interest of the robotic communities involved in unmanned aerial vehicles (UAVs) are increasingly focused on modeling, design and control of multi-rotors vehicles mainly because of the simple rotor mechanics required for flight control. Thanks to their versatility, these platforms are recently becoming a mature technology and their application field spans a wide variety of different tasks. For the real world multi-rotor deployment the robustness to rotorfailure(s) is an essential requirement, therefore such control problem has been widely investigated in the last years.

Given the popularity of quadrotor platforms, a wide literature is available about detection and recovery in case of both partial and complete (see, e.g., [1] and the references within) loss of propeller(s) for such vehicles, guaranteeing position (but not full-orientation) controllability. Nevertheless, recently, several fault-tolerant controllers (FTCs) have been presented also for more complex aerial structures whose propellers number is greater than four. In [2] an adaptive FTC, which relaxes the assumption on control effectiveness

\footnotetext{
${ }^{1}$ LAAS-CNRS, Université de Toulouse, CNRS, Toulouse, France. giulia.michieletto@laas.fr, markus.ryll@laas.fr,antonio.franchi@laas.fr

${ }^{2}$ Dep. of Information Engineering, University of Padova, Padova, Italy.

This work has been partially funded by the European Union's Horizon 2020 research and innovation program under grant agreement No 644271 AEROARMS

During this work Giulia Michieletto has been co-funded by the Eiffel Excellence Scholarship Programme of the the French Ministry of Foreign Affairs and International Development and Markus Ryll has been co-funded by the German Research Foundation (DFG).
}

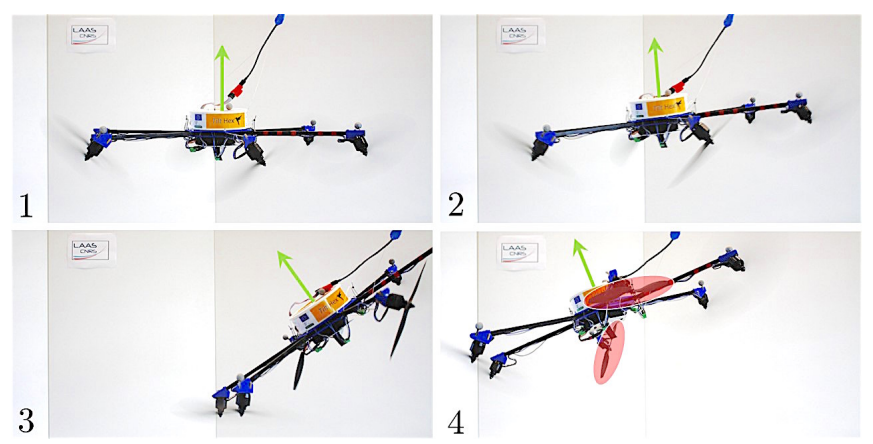

Fig. 1: A real hexarotor recovering from a failure thanks to the analysis and the controller proposed in this paper. The numbers indicate the different phases of the experiment explained in Sec. VI. Notice to the non-spinning propellers marked in the bottomright frame.

matrix, is presented for a six-rotor UAV. This approach is successfully applied to a position (not orientation) tracking problem in the case of one propeller loss. In [3] the authors address the case of two failures in a hexarotor proposing a time delay control method which allows to maintain the platform at a desired altitude with continuous positive pitch and roll attitude command inputs, while control authority is lost on yaw angle that can evolve freely without any bounds. In the recent past, the authors of [4] present a theoretical analysis about the fault-tolerance of a six-rotor vehicle, highlighting the fact that tilting the propellers of a small fixed angle about the frame tangential axis allows to keep the ability to reject disturbance torques in all directions in case of a rotor-failure.

In this work, the rotor-failure issue is faced for the hexarotor platforms described in [4]-[6]. This choice is motivated by the fact that such structures are very popular as they constitute the simplest redundant multi-rotor vehicles. In addition, their dynamics is under-actuated or fully-actuated depending on the directions of the rotors spinning axes. The concept of rotor-failure robustness introduced here and investigated rests upon the capability to still achieve static hovering in case of failure of one propeller. For failure we intend the worst case scenario, in which the propeller is not usable anymore and it is not able to produce any thrust. For static hoverability we instead mean that the platform is able to keep a constant position and a constant orientation without rotating around any axis. Relaxed (dynamic) versions of the hovering concept have been recently introduced in [7]. Those solutions represent a valuable resource as last resort methods to attempt saving the platform from catastrophic damages. However, loosing the capability of controlling the 
full attitude implies less stability, increase of the likelihood of hitting the surrounding environment ${ }^{1}$, impossibility to use some of the exteroceptive sensors, and risk to break some parts in the touch down moment. Static hoverability is instead a feature that would allow a damaged platform to slowly and gently land on the ground. The first part of this work is thus dedicated to the derivation of the algebraic prerequisites for the control matrices which allow for the implementation of a cascaded control suitable to keep a multi-rotor platform with any number of generically oriented rotors in static hover.

The rest of the paper is organized as follows. A generic multi-rotor model is described in Sec. II, while Sec. III shows the derivation of the algebraic conditions for the static hoverability. A control law for static hovering is illustrated in Sec. IV. In Sec. V, a detailed study of the hoverability property is conducted considering a propeller-failure in sixrotor vehicles. Sec. VI reports the principal results of the simulative campaign, while the results of preliminary experiment (see. Fig.1) are available in the multimedia attachment. Main conclusions are drawn in Sec. VII.

\section{Generically Tilted Multi-Rotor}

A large part of aerial platforms described in literature can be ideally modeled as Generically Tilted Multi-Rotors (GTMs). A GTM consists of a rigid body and $n$ lightweight propellers (with mass, gyroscopic effect, and moment of inertia all negligible w.r.t. the body inertial parameters) each spinning about its own axis (including the special cases of all parallel or all different axes). The number $n$ of propellers and the axes mutual orientations determine if the GTM is an under-actuated or full-actuated system [6].

In order to describe the dynamics of a GTM, let us introduce the inertial world frame $\mathscr{F}_{W}=\left\{O_{W},\left(\mathbf{x}_{W}, \mathbf{y}_{W}, \mathbf{z}_{W}\right)\right\}$ and the body frame $\mathscr{F}_{B}=\left\{O_{B},\left(\mathbf{x}_{B}, \mathbf{y}_{B}, \mathbf{z}_{B}\right)\right\}$, whose origin $O_{B}$ coincides with the center of mass (CoM) of the platform. The position of $O_{B}$ in $\mathscr{F}_{W}$ and the orientation of $\mathscr{F}_{B}$ w.r.t. $\mathscr{F}_{W}$ are respectively denoted by the vector $\mathbf{p} \in \mathbb{R}^{3}$ and by the rotation matrix $\mathbf{R} \in S O(3)$, hence the pair $\mathbf{q}=(\mathbf{p}, \mathbf{R}) \in S E(3)$ describes the full-pose of the GTM in $\mathscr{F}_{W}$. In addition, the twist of the platform is indicated by the pair $(\mathbf{v}, \boldsymbol{\omega})$ where $\mathbf{v}=\dot{\mathbf{p}}$ denotes the linear velocity of $O_{B}$ in $\mathscr{F}_{W}$, and $\boldsymbol{\omega}$ is the angular velocity of $\mathscr{F}_{B}$ w.r.t. $\mathscr{F}_{W}$, expressed in $\mathscr{F}_{B}$. Thus, the orientation kinematics is governed by the relation

$$
\dot{\mathbf{R}}=\mathbf{R}[\boldsymbol{\omega}]_{\times},
$$

where $[\cdot]_{\times}$is the map that associates any vector in $\mathbb{R}^{3}$ to its corresponding skew-symmetric matrix belonging to $\mathfrak{s o}(3)$.

Let us define also the frame $\mathscr{F}_{P_{i}}=\left\{O_{P_{i}},\left(\mathbf{x}_{P_{i}}, \mathbf{y}_{P_{i}}, \mathbf{z}_{P_{i}}\right)\right\}$ associated to the $i$-th propeller such that $O_{P_{i}}$ and $\mathbf{z}_{P_{i}}$ coincide with its center and its spinning axis, respectively, while $\mathbf{x}_{P_{i}}$ and $\mathbf{y}_{P_{i}}$ identify its spinning plane. The position of $O_{P_{i}}$ in $\mathscr{F}_{B}$ is indicated as $\mathbf{p}_{i} \in \mathbb{R}^{3}$. The rotation between $\mathbf{z}_{P_{i}}$ and $\mathbf{z}_{B}$ is parametrized through the tilt angles $\alpha_{i}, \beta_{i} \in[-\pi, \pi]$ so that $\mathbf{z}_{P_{i}}=\mathbf{R}_{y}\left(\beta_{i}\right) \mathbf{R}_{x}\left(\alpha_{i}\right) \mathbf{z}_{B}$ (where $\mathbf{R}_{x}, \mathbf{R}_{y}, \mathbf{R}_{z}$ are canonical rotation matrices), $\mathbf{x}_{P_{i}}$ is arbitrarily chosen among the ones

\footnotetext{
${ }^{1}$ In fact, in the recent past a lot of attention has been driven toward the so called aerial physical interaction, see e.g., [8]-[10], in which aerial robots are required to fly and operate very close to the surrounding environment.
}

perpendicular to $\mathbf{z}_{P_{i}}$ and, as a consequence $\mathbf{y}_{P_{i}}=\mathbf{z}_{P_{i}} \times \mathbf{x}_{P_{i}}$. Note that the parameters $\left\{\alpha_{i}, \beta_{i}\right\}_{i=1}^{n}$ allow to discriminate between the cases of all parallel and all differently oriented propeller axes. These quantities are here assumed to be always fixed during the flight thus we do not encompass the case of tiltable-rotor platforms.

The $i$-th propeller exerts in $O_{P_{i}}$ a thrust force and a drag moment on the direction $\mathbf{z}_{i}$, which are expressed in $\mathscr{F}_{B}$ by

$$
\mathbf{f}_{i}=f_{i} \mathbf{z}_{P_{i}}=c_{f_{i}} \omega_{i}^{2} \mathbf{z}_{P_{i}}, \quad \boldsymbol{\tau}_{i}= \pm \tau_{i} \mathbf{z}_{P_{i}}= \pm c_{\tau_{i}} \omega_{i}^{2} \mathbf{z}_{P_{i}},
$$

where $c_{f_{i}}, c_{\tau_{i}}$ are constant parameters related to the $i$-th propeller physical features and $\omega_{i}$ is its spinning rate. The positive (negative) sign in the moment equation (2) refers to clockwise (counterclockwise) rotation direction.

The total control force applied on the platform CoM and the total control moment are expressed as

$$
\begin{aligned}
\mathbf{f}_{c}^{W} & =\mathbf{R} \mathbf{f}_{c}=\mathbf{R} \sum_{i=1}^{n} \mathbf{f}_{i}=\mathbf{R} \mathbf{F}_{1} \mathbf{u}, \\
\boldsymbol{\tau}_{c} & =\sum_{i=1}^{n}\left(\left(\mathbf{p}_{i} \times \mathbf{f}_{i}\right)+\boldsymbol{\tau}_{i}\right)=\mathbf{F}_{2} \mathbf{u},
\end{aligned}
$$

where $\mathbf{u}=\left[\begin{array}{lll}u_{1} & \cdots & u_{n}\end{array}\right]^{\top}=\left[\begin{array}{lll}\omega_{1}\left|\omega_{1}\right| & \cdots & \omega_{n}\left|\omega_{n}\right|\end{array}\right]^{\top} \in \mathbb{R}^{n}$, $\mathbf{F}_{1}, \mathbf{F}_{2} \in \mathbb{R}^{3 \times n}$ are the matrices that map the control input vector $\mathbf{u}$ to the control force and the control moment applied to the platform, respectively. Note that $\mathbf{f}_{c}^{W}$ is expressed in $\mathscr{F}_{W}$ while $\mathbf{f}_{c}$ and $\boldsymbol{\tau}_{c}$ are expressed in $\mathscr{F}_{B}$.

Using the Netwon-Euler equations, and neglecting second order effects such as, e.g., the flapping of the lightweight propellers, the dynamics of a GTM can be expressed as

$$
\left[\begin{array}{c}
m \ddot{\mathbf{p}} \\
\mathbf{J} \boldsymbol{\omega}
\end{array}\right]=-\left[\begin{array}{c}
m g \mathbf{e}_{3} \\
\boldsymbol{\omega} \times \mathbf{J} \boldsymbol{\omega}
\end{array}\right]+\left[\begin{array}{c}
\mathbf{R f}_{c} \\
\boldsymbol{\tau}_{c}
\end{array}\right]=\boldsymbol{\xi}(\boldsymbol{\omega})+\left[\begin{array}{c}
\mathbf{R F}_{1} \\
\mathbf{F}_{2}
\end{array}\right] \mathbf{u},
$$

where $g, m$ and $\mathbf{J} \in \mathbb{R}^{3 \times 3}$ represent the gravitational acceleration, the total mass, and the inertia matrix of the platform.

\section{Static Hoverability Conditions}

We say that a given GTM platform, modeled by (1)-(5), is in static hover when its position and attitude are stably kept constant, i.e., the constant state $(\mathbf{p}, \mathbf{R}, \mathbf{v}, \boldsymbol{\omega})=(\mathbf{0}, \mathbf{R}, \mathbf{0}, \mathbf{0})$ represents a (locally stable) forced equilibrium of (5) for some attitude $\mathbf{R} \in S O(3)$ and some constant input $\mathbf{u}$. A platform in static hover is fully safe w.r.t. the surrounding environment. The same does not hold for a platform in dynamic hovering (see [7]) as explained in the Introduction.

In (1) the dynamics of $\boldsymbol{\omega}$ influences the one of $\mathbf{R}$, which in turn influences through (5) the dynamics of $\mathbf{v}$, and then of p. The one-way nature of this influence chain makes GTMs cascaded dynamical systems, a property that has been used often for controlling these kind of platforms. The main idea is that one first implements, independently from the position/velocity dynamics, an inner control loop that achieves the feedback control of $\boldsymbol{\omega}$ and $\mathbf{R}$ exploiting the control input $\boldsymbol{\tau}_{c}$. Then one considers $\mathbf{R}$ as an additional control input and uses it together with $\mathbf{f}_{c}$, to implement an outer loop for the feedback control of $\mathbf{v}$ and $\mathbf{p}$.

In order to implement the virtual inputs $\boldsymbol{\tau}_{c}$ and $\mathbf{f}_{c}$ in (5) one has to 'invert' the maps $\mathbf{F}_{1}$ and $\mathbf{F}_{2}$ by suitably choosing the real inputs $\mathbf{u}$. The first prerequisite to do so is the fullactuation of the orientation dynamics, i.e., the possibility 
to freely choose $\tau_{c}$ in an sufficiently large open set of $\mathbb{R}^{3}$ containing $\mathbf{0}$. This is equivalent to impose that $\mathbf{F}_{2}$ is full-rank, namely $r k\left(\mathbf{F}_{2}\right)=3$. The second prerequisite is that $\left\|\mathbf{f}_{c}\right\|$ can be chosen independently of $\boldsymbol{\tau}_{c}$. In fact, while $\mathbf{R}$ can change the direction of $\mathbf{f}_{c}^{W}=\mathbf{R} \mathbf{f}_{c}$, it cannot change its norm: the role of $\left\|\mathbf{f}_{c}\right\|$ is then fundamental in order to assign the norm of $\mathbf{f}_{c}^{W}$ independently from $\boldsymbol{\tau}_{c}$. The second prerequisite is ensured if $r k\left(\left[\mathbf{F}_{1}^{\top} \mathbf{F}_{2}^{\top}\right]\right) \geq 4$, which can be recast as the existence of a vector $\mathbf{b}_{2} \in \operatorname{ker}\left(\mathbf{F}_{2}\right) \cap \mathbb{S}^{n-1}$ such that $\mathbf{F}_{1} \mathbf{b}_{2} \neq \mathbf{0}$.

The conditions $\mathbf{u} \in \operatorname{ker}\left(\mathbf{F}_{2}\right)$ and $\mathbf{R F}_{1} \mathbf{u}=m g \mathbf{e}_{3}$ required at the hovering equilibrium are both satisfied if one sets $\mathbf{u}=$ $\delta \mathbf{b}_{2}$ with $\delta=m g /\left\|\mathbf{F}_{1} \mathbf{b}_{2}\right\|$ and $\mathbf{R}$ is such that $\mathbf{R} \mathbf{F}_{1} \mathbf{b}_{2} \propto \mathbf{e}_{3}$. In this way any vector $\mathbf{b}_{2}$ satisfying the second prerequisite geometrically represents a line in the input space $\mathbb{R}^{n}$, along which a command $\mathbf{u}$ can lie to keep the platform hovering. The working point along this line is decided by the $m$ and the propellers characteristics and arrangement, through the matrices $\mathbf{F}_{1}$ and $\mathbf{F}_{2}$ (the former appears directly in $\left\|\mathbf{F}_{1} \mathbf{b}_{2}\right\|$ and the latter appears indirectly, since $\mathbf{b}_{2} \in \operatorname{ker}\left(\mathbf{F}_{2}\right)$ ).

In normal working condition the $i$-th propeller spinning rate $\omega_{i}$ must belong to a certain set of feasible frequencies, namely $u_{i} \in U_{i}, i=1, \ldots, n$. The third prerequisite is then that the working point input commands corresponding to a hovering equilibrium lie 'inside enough' the allowable input set $U_{1} \times \ldots \times U_{n}$, such that additional input maneuverability is left for controlling the attitude and compensating the position errors. One can recast the third prerequisite on the vector $\mathbf{b}_{2}$ imposing that $\delta \mathbf{b}_{2} \in H_{1} \times \ldots \times H_{n}$, where $H_{1} \subset U_{1}, \ldots, H_{n} \subset U_{n}$ are the sets of conservative hovering commands.

The third prerequisite can be slightly relaxed assuming that a subset of rotors with indexes in a set $\mathscr{C} \subset \mathscr{N}=$ $\{1, \ldots, n\}$ is not used for dynamic control but is just kept at a constant spinning rate for the whole duration of the flight, i.e., $u_{j}(t)=u_{j}^{*} \forall j \in \mathscr{C}, \forall t \geq t_{0}$. In this case the third requirement becomes less stringent, in fact it can be tolerated that $\delta \mathbf{b}_{2, j}=u_{j}^{*}$ belongs to the larger set $U_{j}$ (instead of $H_{j}$ ) for any $j \in \mathscr{C}$, where $\mathbf{b}_{2, j}$ represents the $j$-th entry of $\mathbf{b}_{2}$. On the other side by doing so only the control inputs $u_{i}$ with $i \in \mathscr{N} \backslash \mathscr{C}$ can be used for dynamic control, which means that the first and second prerequisites must be changed accordingly. Denoting with $\mathbf{F}_{1}^{\mathscr{C}}, \mathbf{F}_{2}^{\mathscr{C}}$, and $\mathbf{b}_{2}^{\mathscr{C}}$ the matrices (vector) obtained from $\mathbf{F}_{1}, \mathbf{F}_{2},\left(\mathbf{b}_{2}\right)$ by removing the columns (entries) whose index is in $\mathscr{C}$ we have in conclusion following conditions.

Static Hoverability Conditions (SHCs). A GTM is said statically hoverable if it exists $\mathscr{C} \subset \mathscr{N}$ and a set of values $\left\{u_{j}^{*} \in U_{j}\right\}_{j \in \mathscr{C}}$ such that the following conditions are satisfied

1) $r k\left(\mathbf{F}_{2}^{\mathscr{C}}\right)=3$

2) $\exists \mathbf{b}_{2} \in \operatorname{ker}\left(\mathbf{F}_{2}\right) \cap \mathbb{S}^{n-1}$ such that

a) $\mathbf{F}_{1}^{\mathscr{C}} \mathbf{b}_{2}^{\mathscr{C}} \neq \mathbf{0}$

b) denoting with $\delta=m g /\left\|\mathbf{F}_{1} \mathbf{b}_{2}\right\|$ it is

i) $\delta \mathbf{b}_{2, j}=u_{j}^{*} \forall j \in \mathscr{C}$ and

ii) $\delta \mathbf{b}_{2, j} \in H_{j}$ for any other $j$.

Proposition 1. A straightforward consequence of the SHCs is that a GTM can be statically hoverable only if $n-|\mathscr{C}| \geq 4$.

\section{HOVER CONTROL}

In this section we propose a controller to stably keep a statically hoverable GTM in static hover. It is worth to notice that statically hoverable platforms represent a much larger class of UAVs than the typical ones encountered in the literature, such as, e.g., collinear propeller or fully-actuated platforms. Therefore the main contribution of this section is to provide a control law for such larger class of vehicles.

Formally, the goal of the proposed controller is to keep the platform at a given constant reference position $\mathbf{p}_{r} \in \mathbb{R}^{3}$ and with a constant attitude that is as close as possible to a given reference one defined by a constant given rotation matrix $\mathbf{R}_{r} \in S O(3)$. The controller is composed by an outer control loop (position controller) computing the reference control force $\mathbf{f}_{r}$ and the desired orientation $\mathbf{R}_{d}$, which is passed to an inner control loop (attitude controller) which outputs the reference control moment $\tau_{r}$. The actual input is computed by a wrench mapper that determines $\mathbf{u}$ so that $\boldsymbol{\tau}_{c}=\mathbf{F}_{2} \mathbf{u}=\boldsymbol{\tau}_{r}$ and, at least, the projection of the control force $\mathbf{f}_{c}=\mathbf{F}_{1} \mathbf{u}$ along a certain direction is equal to the norm of $\mathbf{f}_{r}$.

The key feature of the control strategy is indeed the identification of a preferential direction in the force space, denoted with $\mathbf{d}_{*} \in \operatorname{Im}\left(\mathbf{F}_{1}\right) \cap \mathbb{S}^{2}$, along which the intensity of the control force can be assigned independently from $\tau_{c}$. This is equivalent to find a vector $\mathbf{d}_{*} \in \operatorname{Im}\left(\mathbf{F}_{1}\right) \cap \mathbb{S}^{2}$ such that $r k\left(\left[\mathbf{F}_{1}^{\top} \mathbf{d}_{*} \mathbf{F}_{2}^{\top}\right]\right) \geq 4$. In fact, if that rank inequality is satisfied, then $\mathbf{d}_{*}^{\top} \mathbf{F}_{1} \mathbf{u}$ can be arbitrarily assigned regardless of the fact that it also has to be $\mathbf{F}_{2} \mathbf{u}=\boldsymbol{\tau}_{r}$. Assuming that the SHCs are fulfilled, then it is easy to check that a reasonable and viable choice is to take exactly $\mathbf{d}_{*}=\mathbf{F}_{1} \mathbf{b}_{2} /\left\|\mathbf{F}_{1} \mathbf{b}_{2}\right\|$. Notice that $\mathbf{d}_{*}$ is not necessarily parallel to $\mathbf{z}_{B}$.

The structure of the proposed controller consists of the three main blocks here described.

1) Position Controller: Let be given a constant reference position $\mathbf{p}_{r} \in \mathbb{R}^{3}$, then a good choice to steer the position error $\mathbf{e}_{p}=\mathbf{p}-\mathbf{p}_{r}$ to zerois to apply to the vehicle the force

$$
\mathbf{f}_{r}=m g \mathbf{e}_{3}-\mathbf{K}_{p} \mathbf{e}_{p}-\mathbf{K}_{v} \mathbf{v},
$$

which is a simple PD + gravity compensation control with positive definite diagonal gain matrices $\mathbf{K}_{p}, \mathbf{K}_{v} \in \mathbb{R}^{3 \times 3}$.

The fundamental idea behind the proposed controller is to obtain $\mathbf{f}_{c}=\mathbf{f}_{r}$ by rotating the platform in such a way that the preferential direction $\mathbf{d}_{*}$ is aligned with $\mathbf{f}_{r}$. In fact in this way, both the direction and the intensity of $\mathbf{f}_{c}$ can be made equal to ones of $\mathbf{f}_{r}$ regardless of the implemented moment $\boldsymbol{\tau}_{c}$. To do so the position controller computes the desired orientation $\mathbf{R}_{d} \in S O(3)$. This carries out the alignment of the preferential direction to the direction of the required control force, i.e., $\mathbf{R}_{d}$ is such that $\mathbf{R}_{d} \mathbf{d}_{*}=\mathbf{f}_{r} /\left\|\mathbf{f}_{r}\right\|$, and at the same time tries to minimize the distance from the reference orientation $\mathbf{R}_{r}$.

The desired orientation $\mathbf{R}_{d}$ is univocally determined among the infinite possible solutions by first computing the minimal rotation $\mathbf{R}_{b} \in S O(3)$ that aligns the preferential direction and $\mathbf{z}_{W}$. This is done through the Rodrigues' rotation formula. Then, a matrix $\mathbf{R}_{w} \in S O(3)$ is defined in order to fix one of the infinite rotations that align $\mathbf{z}_{W}$ to $\mathbf{f}_{r} /\left\|\mathbf{f}_{r}\right\|$. To do so, the requirement on minimal distance from $\mathbf{R}_{r}$ is exploited 
by setting $\mathbf{R}_{w}=\left[\left(\left(\mathbf{r}_{3} \times \mathbf{r}_{1)} \times \mathbf{r}_{3}\right)\left(\mathbf{r}_{3} \times \mathbf{r}_{1}\right) \mathbf{r}_{3}\right]\right.$, where $\mathbf{r}_{1}$ is to the first column of $\mathbf{R}_{r}$ and $\mathbf{r}_{3}=\mathbf{f}_{r} /\left\|\mathbf{f}_{r}\right\|$. The desired orientation is univocally determined by $\mathbf{R}_{d}=\mathbf{R}_{w} \mathbf{R}_{b}$.

2) Attitude Controller: To let $\mathbf{R}$ track $\mathbf{R}_{d}$, the standard geometric control moment is computed as

$$
\boldsymbol{\tau}_{r}=\boldsymbol{\omega} \times \mathbf{J} \boldsymbol{\omega}-\mathbf{K}_{R} \mathbf{e}_{R}-\mathbf{K}_{\omega} \boldsymbol{\omega},
$$

where the positive definite diagonal gain matrices $\mathbf{K}_{R}, \mathbf{K}_{\omega} \in$ $\mathbb{R}^{3 \times 3}$ allow to tune the effect of the rotation tracking error $\mathbf{e}_{R}$ and the angular velocity feedback term, respectively. Specifically, the rotation tracking error is chosen to be

$$
\mathbf{e}_{R}=\frac{1}{2}\left(\mathbf{R}_{d}^{\top} \mathbf{R}-\mathbf{R}^{\top} \mathbf{R}_{d}\right)^{\vee}
$$

where the operator $[\cdot]^{\vee}$ describes the map from $\mathfrak{s o}(3)$ to $\mathbb{R}^{3}$.

3) Wrench Mapper: The proposed control law prioritizes the fulfillment of the required control torque, namely to obtain $\boldsymbol{\tau}_{c}=\boldsymbol{\tau}_{r}$. To do so the actual control input is designed as the sum of two terms, $\mathbf{u}_{\tau}$ and $\mathbf{u}_{f}$, where $\mathbf{u}_{\tau}=\mathbf{F}_{2}^{\dagger} \boldsymbol{\tau}_{r}$ and $\mathbf{u}_{f}=\mathbf{b}_{2} c$. The value of the parameter $c \in \mathbb{R}$ is set requiring that the total force applied to the body, i.e., $\mathbf{R F}_{1} \mathbf{u}$, is made as close as possible to $\mathbf{f}_{r}$, thus solving

$$
\min _{c}\left\|\mathbf{R F}_{1}\left(\mathbf{F}_{2}^{\dagger} \boldsymbol{\tau}_{r}+\mathbf{b}_{2} c\right)-\mathbf{f}_{r}\right\|^{2},
$$

whose solution in closed form is obtained through the simple vector projection using the formula

$$
c=\frac{\left(\mathbf{f}_{r}-\mathbf{R} \mathbf{F}_{1} \mathbf{F}_{2}^{\dagger} \boldsymbol{\tau}_{r}\right)^{T} \mathbf{R} \mathbf{F}_{1} \mathbf{b}_{2}}{\left\|\mathbf{R} \mathbf{F}_{1} \mathbf{b}_{2}\right\|^{2}}=\frac{\left(\mathbf{f}_{r}-\mathbf{R} \mathbf{F}_{1} \mathbf{F}_{2}^{\dagger} \boldsymbol{\tau}_{r}\right)^{T} \mathbf{R} \mathbf{d}_{*}}{\left\|\mathbf{F}_{1} \mathbf{b}_{2}\right\|} .
$$

As a consequence, the total input control law $\mathbf{u}$ results to be

$$
\mathbf{u}=\mathbf{F}_{2}^{\dagger} \boldsymbol{\tau}_{r}+\mathbf{b}_{2}\left(\mathbf{f}_{r}-\mathbf{R} \mathbf{F}_{1} \mathbf{F}_{2}^{\dagger} \boldsymbol{\tau}_{r}\right)^{T} \mathbf{R} \mathbf{d}_{*}\left\|\mathbf{F}_{1} \mathbf{b}_{2}\right\|^{-1} .
$$

Note that as long as $\mathbf{R} \mathbf{d}_{*}$ is kept parallel to $\mathbf{f}_{r}$ then $\boldsymbol{\tau}_{r}=\mathbf{0}$, and therefore we obtain that the total force is $\mathbf{R F}_{1} \mathbf{b}_{2} \frac{\mathbf{f}_{r}^{T} \mathbf{R d}_{*}}{\left\|\mathbf{F}_{1} \mathbf{b}_{2}\right\|}=$ $\mathbf{R d}_{*}{ }_{r}^{T} \mathbf{R d}_{*}=\mathbf{f}_{r}$ as it should be.

Thanks to control continuity, the control input is kept feasible at least in a neighborhood of the equilibrium due to the fact that the commands at the equilibrium are designed to be well inside the feasibility set by virtue of the third prerequisite.

\section{Hexarotor Robustness Analysis}

In this section we introduce and investigate the concept of rotor-failure robustness for a hexarotor GTM, i.e., having 6 propellers, defined as its capability to still achieve static hover in the case a rotor fails and stops to spin. We consider the most common case in which $U_{i}=[0, \bar{u}]$ and $H_{i}=[\underline{h}, \bar{h}]$, where $0<\underline{h}<\bar{h}<\bar{u}$ for $i \in \mathscr{S}=\{1, \ldots, 6\}$, and in which $c_{\tau_{i}}=c_{\tau}, c_{f_{i}}=c_{f}$ for $i=1, \ldots, 6$.

Definition 1. In the following, 'the $k$-th rotor is failed' means that it cannot spin anymore $\left(\omega_{k}=u_{k} \equiv 0\right)$. A rotor that is not failed is healthy.

Definition 2. A hexarotor GTM is said to be $\{k\}$-loss robust, with $k \in \mathscr{S}$ if the obtained 'penta'-rotor GTM considering the novel set $\mathscr{N}=\mathscr{S} \backslash\{k\}$ is still statically hoverable.

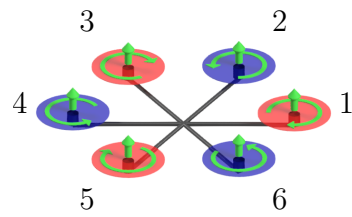

(a) Collinear Hexarotor

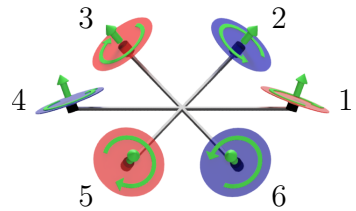

(b) Tilted Hexarotor
Fig. 2: Platforms considered in the rotor-failure robustness analysis: blue and red discs correspond to rotors with concordant and opposing thrust force and drag moment respectively.

Definition 3. A hexarotor is fully robust if it is $\{k\}$-loss robust for any $k \in \mathscr{S}$.

Definition 4. A hexarotor that is not fully robust is partially robust if it is $\{k\}$-loss robust for at least one $k \in \mathscr{S}$.

Definition 5. A hexarotor that is neither fully robust nor partially robust is fully vulnerable.

Let us denote by $\mathbf{A}_{1}, \mathbf{A}_{2} \in \mathbb{R}^{3 \times 6}$ the control force and moment input matrices of the considered hexarotor GTM (i.e., $\mathbf{F}_{1}$ and $\mathbf{F}_{2}$ in (5), respectively). In addition, we indicate as ${ }^{k} \mathbf{A}_{1}$ and ${ }^{k} \mathbf{A}_{2}$ the matrices obtained from $\mathbf{A}_{1}$ and $\mathbf{A}_{2}$ by removing the $k$-th column and by ${ }^{k} \mathbf{A}_{1}^{j}$ and ${ }^{k} \mathbf{A}_{2}^{j}$ the matrices obtained from ${ }^{k} \mathbf{A}_{2}^{j}$ by removing both the $k$-th and the $j$-th column.

In the following, we compare the robustness properties of the hexarotor platforms with parallel propeller axes (Collinear Hexarotor, Fig. 2a) w.r.t. the case in which the rotor axes are differently oriented (Tilted Hexarotor, Fig. 2b).

\section{A. Collinear Hexarotor}

Contrarily to the common sense, which associates robustness with redundancy (an hexarotor has six rotors while a quadrotor only four), we have the following clear result.

Proposition 2. The collinear hexarotor (having $\alpha_{i}=\beta_{i}=0$ for $i=1, \ldots, 6)$ is fully vulnerable.

Proof. Due to Prop. 1 the set $\mathscr{C}$ can either be $\emptyset$ or $\{j\}$. Let us consider the first case first, i.e., $\mathbf{F}_{2}^{\mathscr{C}}={ }^{k} \mathbf{A}_{2} \in \mathbb{R}^{3 \times 5}$. The $k$-th row of Tab. I reports the null-space generating vectors and the rank of ${ }^{k} \mathbf{A}_{2}$ for $k \in \mathscr{S}$. It is clear to see that any linear combination of the generating vectors has a 0 in correspondence of the entry $l=k+3$ (for $k=1,2,3$ ) or $l=k-3$ (for $k=4,5,6$ ). This implies that $\mathbf{b}_{2, l}=0 \notin H_{l}$.

Let us now consider the case in which $\mathscr{C}=\{l\}$. The only possible value is $u_{l}^{*}=0$ (because it must hold that $\mathbf{b}_{2} \in$ $\operatorname{ker}\left(\mathbf{A}_{2}\right)$ ). This corresponds to switching off the rotor opposed to the failed one. The $k$-th row of Tab. II reports the nullspace generating vectors and the rank of ${ }^{k} \mathbf{A}_{2}^{l} \in \mathbb{R}^{3 \times 4}$. Also in this case the SHCs are not verified, this time because $r k\left({ }^{k} \mathbf{A}_{2}^{l}\right)=2$ for any $k \in \mathscr{S}$. Finally, consider the case in which $\mathscr{C}=\{j\}$ with $j \neq l$. Whatever the value is chosen for $u_{j}^{*}$, it holds (like in the $\mathscr{C}=\emptyset$ case) that $\mathbf{b}_{2, l}=0 \notin H_{l}$.

Therefore we can conclude that it is impossible to fulfill the SHCs for a collinear hexarotor GTM if any of its six propeller fails. 


\begin{tabular}{|c|c|c|c|c|c|c|c|}
\hline & \multicolumn{6}{|c|}{ null-space generators } & rank \\
\hline \multirow{2}{*}{${ }^{1} \mathbf{A}_{2}$} & (foil) & {$[1$} & 0 & 0 & 1 & $0]^{\top}$ & \multirow{2}{*}{3} \\
\hline & (tail) & {$[0$} & 1 & 0 & 0 & $1]^{\top}$ & \\
\hline \multirow{2}{*}{${ }^{2} \mathbf{A}_{2}$} & {$[1$} & (f il) & 0 & 1 & 0 & $0]^{\top}$ & \multirow{2}{*}{3} \\
\hline & 0 & (fail) & 1 & 0 & 0 & $1]^{\top}$ & \\
\hline \multirow{2}{*}{${ }^{3} \mathbf{A}_{2}$} & {$[1$} & 0 & \multirow{2}{*}{ (fail) } & 1 & 0 & $0]^{\top}$ & \multirow{2}{*}{3} \\
\hline & {$[0$} & 1 & & 0 & 1 & $0]^{\top}$ & \\
\hline \multirow{2}{*}{${ }^{4} \mathbf{A}_{2}$} & {$[0$} & 1 & 0 & (foil) & 1 & $0]^{\top}$ & \multirow{2}{*}{3} \\
\hline & {$[0$} & 0 & 1 & (fail) & 0 & $1]^{\top}$ & \\
\hline \multirow{2}{*}{${ }^{5} \mathbf{A}_{2}$} & {$[1$} & 0 & 0 & 1 & foil & $0]^{\top}$ & \multirow{2}{*}{3} \\
\hline & {$[0$} & 0 & 1 & 0 & (fail) & $1]^{\top}$ & \\
\hline \multirow{4}{*}{${ }^{6} \mathbf{A}_{2}$} & {$[1$} & 0 & 0 & 1 & $0]^{\top}$ & \multirow{2}{*}{ (fail) } & \multirow{2}{*}{3} \\
\hline & {$[0$} & 1 & 0 & 0 & $1]^{\top}$ & & \\
\hline & 1 & 2 & 3 & 4 & 5 & 6 & \\
\hline & \multicolumn{6}{|c|}{ propellers } & \\
\hline
\end{tabular}

TABLE I: Rank and null-space analysis for ${ }^{k} \mathbf{A}_{2}$.

\begin{tabular}{|c|c|c|c|c|c|c|c|}
\hline & \multicolumn{6}{|c|}{ null-space generators } & rank \\
\hline \multirow{2}{*}{${ }^{1} \mathbf{A}_{2}^{4}$} & (fail) & {$[1$} & 0 & (off) & 1 & $0]^{\top}$ & 2 \\
\hline & (Iail) & {$[0$} & 1 & (OII) & 0 & $1]^{\top}$ & 2 \\
\hline \multirow{2}{*}{${ }^{2} \mathbf{A}_{2}^{5}$} & {$[1$} & (fail) & 0 & 1 & & $\mathrm{cl}^{\top}$ & \\
\hline & {$[0$} & (Iail) & 1 & 0 & (olf) & $1]^{\top}$ & 2 \\
\hline \multirow{2}{*}{${ }^{3} \mathbf{A}_{2}^{6}$} & {$[1$} & 0 & \multirow{2}{*}{ (fail) } & 1 & $0]^{\top}$ & \multirow{2}{*}{ (off) } & \multirow{2}{*}{2} \\
\hline & {$[0$} & 1 & & 0 & $1]^{\top}$ & & \\
\hline \multirow{2}{*}{${ }^{4} \mathbf{A}_{2}^{1}$} & (off) & {$[1$} & 0 & \multirow{2}{*}{ (fail) } & 1 & $0]^{\top}$ & \multirow{2}{*}{2} \\
\hline & (OII) & {$[0$} & 1 & & 0 & $1]^{\top}$ & \\
\hline \multirow{2}{*}{${ }^{5} \mathbf{A}_{2}^{2}$} & [ 1 & (off) & 0 & 1 & (fail) & $0]^{\top}$ & \multirow{2}{*}{2} \\
\hline & {$[0$} & (OII) & 1 & 0 & (Iail) & $1]^{\top}$ & \\
\hline \multirow{4}{*}{${ }^{6} \mathbf{A}_{2}^{3}$} & {$[1$} & 0 & \multirow{2}{*}{ (off) } & 1 & $0]^{\top}$ & (fail) & \multirow{2}{*}{2} \\
\hline & {$[0$} & 1 & & 0 & $1]^{\top}$ & (Iail) & \\
\hline & 1 & 2 & 3 & 4 & 5 & 6 & \\
\hline & \multicolumn{6}{|c|}{ propellers } & \\
\hline
\end{tabular}

TABLE II: Rank and null-space analysis for ${ }^{k} \mathbf{A}_{2}^{l}$, where $l$ corresponds to the propeller opposed to the $k$-th one.

\section{B. Tilted Hexarotor}

Let us now consider the case in which the rotors are non collinear, and let us focus on the case in which $\alpha_{i}=$ $(-1)^{i-1} \alpha$ and $\beta_{i}=\beta, i=, 1 \ldots, 6$, where $\alpha, \beta \in[-\pi / 4, \pi / 4]$. We distinguish three different sets (cases) of configuration:

(i) rotors tilted only about their $\mathbf{x}_{P_{i}}$-axis $(\alpha \neq 0, \beta=0)$;

(ii) rotors tilted only about the $\mathbf{y}_{P_{i}}$-axis $(\alpha=0, \beta \neq 0)$;

(iii) rotors tilted both about $\mathbf{x}_{P_{i}}$-axis and $\mathbf{y}_{P_{i}}$-axis $(\alpha, \beta \neq 0)$.

In Sec. V-A we have seen that a major obstacle for the satisfaction of the SHCs is the fact that $r k\left({ }^{k} \mathbf{A}_{2}^{l}\right)=2$ for any $k, l \in \mathscr{S}$ defined as before. In fact if we consider, e.g., $k=3$ (and consequently $l=k+3=6$ ) we obtain

$$
{ }^{3} \mathbf{A}_{2}^{6}=c_{\tau}\left[\begin{array}{cccc}
0 & \frac{\sqrt{3}}{2} r & 0 & -\frac{\sqrt{3}}{2} r \\
-r & -\frac{1}{2} r & r & \frac{1}{2} r \\
1 & -1 & -1 & 1
\end{array}\right] .
$$

with $r=\frac{c_{f}}{c_{\tau}} \ell$ where $\ell$ is the distance between $O_{B}$ and $O_{P_{i}}$ for all propellers. It is straightforward to verify that $r k\left({ }^{3} \mathbf{A}_{2}^{6}\right)=2$, in accordance with Tab. II. A similar structure appears for different $k$ 's. This highlights the major difference between a quadrotor and a failed collinear hexarotor: in the latter case the component of the control moment along the $\mathbf{z}_{B}$ axis is linearly dependent to the other two components along $\mathbf{x}_{B}$ and $\mathbf{y}_{B}$. This happens because the direction of rotation of the four propellers is not alternated as it is instead in the quadrotor case (as clearly visible in Fig. 2a imagining to remove any pair of opposite propellers).

1) Role of $\beta$ (full-rankness): If all propellers are inward (outward) tilted by an angle $\beta>0$ and $\alpha=0$, then all thrust

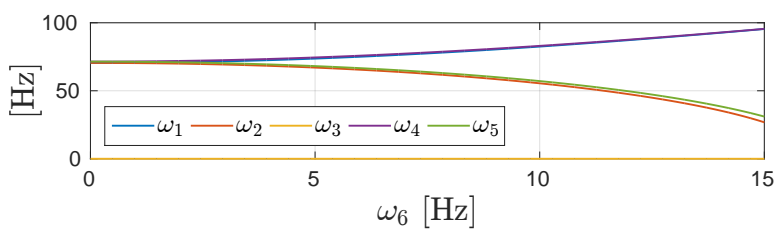

Fig. 3: Spinning rates at hovering equilibrium when $\omega_{6}$ changes in $[0,15] \mathrm{Hz}$.

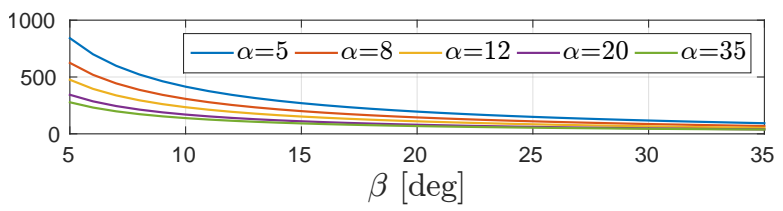

Fig. 4: Condition number of matrix ${ }^{k} \mathbf{A}_{2}^{l}$ w.r.t. $\beta$ angle for different values (in degrees) of $\alpha$ angle.

vectors are inward (outward) tilted, while the drag vectors are inward tilted for the rotors whose drag sign is positive and they are outward tilted for the rotors whose drag sign is negative. As a consequence, it occurs that

$$
{ }^{3} \mathbf{A}_{2}^{6}(\beta)=c_{\tau}\left[\begin{array}{cccc}
s \beta & -\frac{1}{2} s \beta+\frac{\sqrt{3}}{2} r c \beta & s \beta & -\frac{1}{2} s \beta-\frac{\sqrt{3}}{2} r c \beta \\
-r c \beta-\frac{\sqrt{3}}{2} s \beta-\frac{1}{2} r c \beta & r c \beta & -\frac{\sqrt{3}}{2} s \beta+\frac{1}{2} r c \beta \\
c \beta & -c \beta & -c \beta & c \beta
\end{array}\right],
$$

where $s \beta=\sin \beta$ and $c \beta=\cos \beta$ for compactness. It is possible to see that $r k\left({ }^{3} \mathbf{A}_{2}^{6}(\beta \neq 0)\right)=3$ and that in general $r k\left({ }^{k} \mathbf{A}_{2}^{l}(\beta \neq 0)\right)=3$ for any $k \in \mathscr{S}$. The condition $\beta \neq 0$ ensures a decoupling between the component of the control moment along the $\mathbf{z}_{B}$ axis and ones along $\mathbf{x}_{B}$ and $\mathbf{y}_{B}$.

Another effect of choosing $\beta \neq 0$ is that the $l$-th component of a generic vector in $\operatorname{ker}\left({ }^{k} \mathbf{A}_{2}\right)$ is not anymore necessarily zero (even though it is typically a 'small' number compared to the other entries). Therefore it is not mandatory to select $u_{l}^{*}=0$ as in the collinear case. In Fig. 3 we plot the spinning rates required at the hovering equilibrium when the propeller $k=3$ is failed (i.e, $\omega_{3}=0 \mathrm{~Hz}$ ) for different values of $\omega_{l}^{*}, l=k+3=6$ and $\beta=25 \mathrm{deg}$. It is clear as $u_{l}^{*}=0$ represents in this case the best solution in order to balance the control effort.

Any nonzero value of $\beta$ ensures the full-rankness of the control moment input matrix however a very small value means that the matrix is close to loose full-rankness (i.e., it has a large condition number). On the other side, a large value of $\beta$ implies energy dissipation due to internal forces and unbalanced control inputs at the hovering equilibrium. A good tradeoff has to be found between the two needs.

2) Role of $\alpha$ (improved condition number): The parameter $\alpha$ is not essential to ensure propeller-failure robustness, in fact, when $\beta=0$ and $\alpha \neq 0$ (case (ii)), the results are the same as described for the collinear hexarotor $\left(r k\left({ }^{k} \mathbf{A}_{2}^{l}\right)=2\right)$. On the other side, when $\beta \neq 0$, a value of alpha $\alpha>0$ lets the condition number of the matrix ${ }^{l} \mathbf{A}_{2}^{k}$ decrease much more rapidly when $\beta$ increase, as it can be seen in Fig. 4 .

We can conclude this section with the following summarizing result.

Proposition 3. A tilted hexarotor is fully vulnerable in case (i), and it is fully robust if $\beta \neq 0$, i.e., in cases (ii) and (iii). 


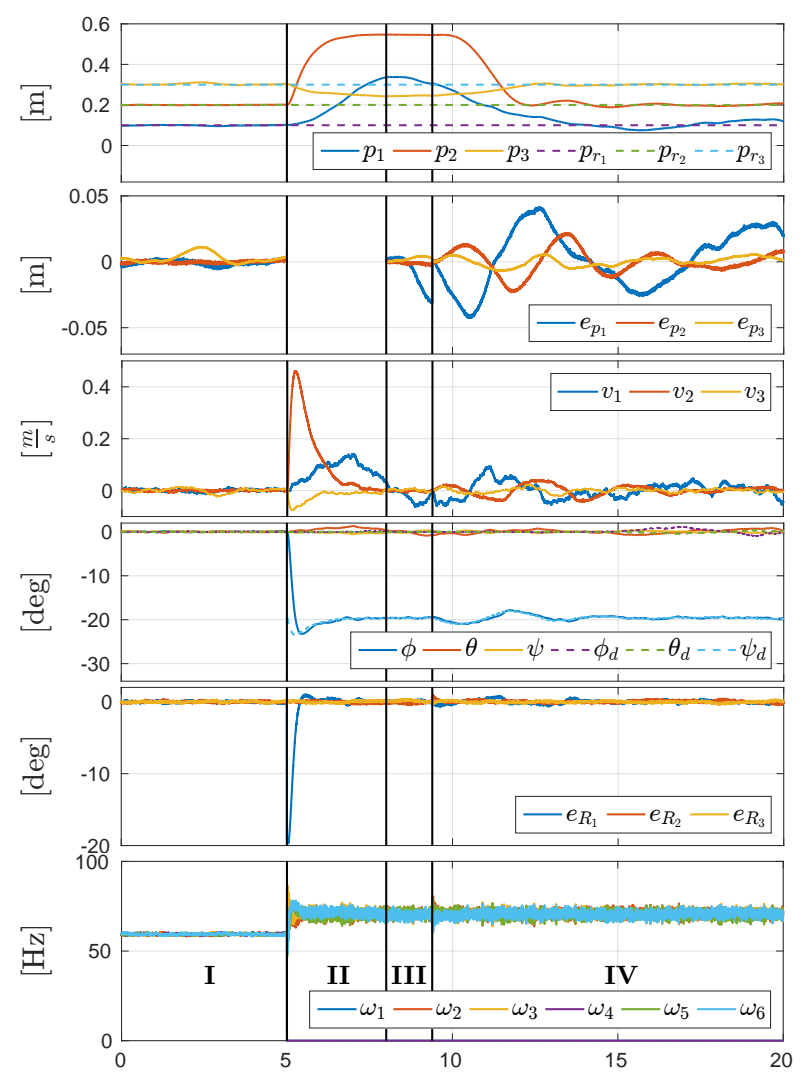

Fig. 5: Non-ideal simulation of the proposed controller in the case of a single failed motor for a GTH having $\alpha=35 \mathrm{deg}$ and $\beta=10$ deg.

\section{Simulations Results}

In this section we present the main simulation results for a hexarotor with $\alpha=35 \mathrm{deg}$ and $\beta=10 \mathrm{deg}$. Specifically, the controller explained in Sec. IV is tested in the case of a rotor-failure. The model presented in (5) has been extended by several real world effects to increase the reliability.

- Position and orientation feedback and their derivatives are loaded with time delay $t_{f}=12 \mathrm{~ms}$, sensor noise according to Tab. III, and a reduced sampling frequency of $100 \mathrm{~Hz}$ while the controller runs at $500 \mathrm{~Hz}$.

- The ESC controller driving the motors is modeled by quantizing the desired $\mathbf{u}$ in (11) resembling a 10 bit discretization in the feasible motor speed between $0 \mathrm{~Hz}$ and $120 \mathrm{~Hz}$. The motor-propeller combination is modeled as a first order transfer function $\left(G(s)=\frac{10}{0.05 s+10}\right)$ and speed-dependent noise (see Tab. III).

In the simulated scenarios the vehicle shall hover at a predefined spot $\mathbf{p}_{r}$ and $\mathbf{R}_{r}$ fulfilling a surveillance task (phase $I$ ). At time $t=5 \mathrm{~s}$ we model the failure of a single rotor and utilize the controller presented in Sec. IV to recover from this threatening situation. Therefore we first set the gain matrix $\mathbf{K}_{p}=\mathbf{0}_{3 \times 3}$ in (6) for a certain time (phase II). In this phase the orientation stabilizes and the velocity error decreases. We then increase $\mathbf{K}_{p}$ using the current position in that time instance as future desired position (phase III). Finally when the norm of the current velocity decreases under a threshold we determine a new trajectory from the current position to the original hovering position to return back (phase IV).

\begin{tabular}{|c|c|c|c|c|}
\hline $\mathbf{p}$ & $\mathbf{v}$ & $\mathbf{R}$ & $\omega$ & $\mathbf{u}$ \\
\hline $6.4 \mathrm{e}-04$ & $1.4 \mathrm{e}-03$ & $1.2 \mathrm{e}-03$ & $2.7 \mathrm{e}-03$ & $0-0.32$ \\
\hline
\end{tabular}

TABLE III: Standard deviation of noise added in the simulations.

The results of the simulation are reported in Fig. 5. The position and orientation error in phase $I$ is negligible. At time $t=5 \mathrm{~s}$, when the rotor-failure occurs, the opposing propeller is switched off and the controller asks for a new hovering orientation (see fourth plot in Fig. 5). Within $\approx 3 \mathrm{~s}$ a negligible velocity is achieved. In phase IV the platform successfully returns to its original position.

To better understand the achieved simulation results the authors suggest referring to the multimedia attachment.

\section{CONCLUSion AND Future Work}

In this work we have derived the algebraic conditions that allow to implement a cascaded controller to keep a Generically Tilted Multi-Rotor platform in static hover. A suitable control law has then been designed and its validity has been proved by simulation results. We have finally conducted a deep analysis on the hoverability capabilities of hexarotor platforms when a rotor fails and the rotor spinning axes are parallel or generically oriented. In future, the validity of the proposed controller will be tested on a real scenario, and a formal proof of its convergence will be provided.

\section{REFERENCES}

[1] M. W. Mueller and R. D'Andrea, "Stability and control of a quadrocopter despite the complete loss of one, two, or three propellers," in 2014 IEEE Int. Conf. on Robotics and Automation, Hong Kong, China, May 2014, pp. 45-52.

[2] G. P. Falconí and F. Holzapfel, "Adaptive fault tolerant control allocation for a hexacopter system," in 2016 American Control Conference, Boston, MA, May 2016, pp. 6760-6766.

[3] J. Lee, H. S. Choi, and H. Shim, "Fault tolerant control of hexacopter for actuator faults using time delay control method," International Journal of Aeronautical and Space Sciences, vol. 17, no. 1, pp. 5463, 2016.

[4] J. I. Giribet, R. S. Sanchez-Pena, and A. S. Ghersin, "Analysis and design of a tilted rotor hexacopter for fault tolerance," IEEE Trans. on Aerospace and Electronic System, vol. 52, no. 4, pp. 1555-1567, 2016.

[5] M. Ryll, D. Bicego, and A. Franchi, "Modeling and control of FASTHex: a fully-actuated by synchronized-tilting hexarotor," in 2016 IEEE/RSJ Int. Conf. on Intelligent Robots and Systems, Daejeon, South Korea, Oct. 2016, pp. 1689-1694.

[6] S. Rajappa, M. Ryll, H. H. Bülthoff, and A. Franchi, "Modeling, control and design optimization for a fully-actuated hexarotor aerial vehicle with tilted propellers," in 2015 IEEE Int. Conf. on Robotics and Automation, Seattle, WA, May 2015, pp. 4006-4013.

[7] M. W. Mueller and R. D'Andrea, "Relaxed hover solutions for multicopters: Application to algorithmic redundancy and novel vehicles," The International Journal of Robotics Research, vol. 35, no. 8, pp. 873-889, 2016.

[8] B. Yüksel, C. Secchi, H. H. Bülthoff, and A. Franchi, "A nonlinear force observer for quadrotors and application to physical interactive tasks," in 2014 IEEE/ASME Int. Conf. on Advanced Intelligent Mechatronics, Besançon, France, Jul. 2014, pp. 433-440.

[9] —_ "Reshaping the physical properties of a quadrotor through IDA$\mathrm{PBC}$ and its application to aerial physical interaction," in 2014 IEEE Int. Conf. on Robotics and Automation, Hong Kong, China, May. 2014 pp. 6258-6265.

[10] G. Gioioso, M. Ryll, D. Prattichizzo, H. H. Bülthoff, and A. Franchi, "Turning a near-hovering controlled quadrotor into a 3D force effector," in 2014 IEEE Int. Conf. on Robotics and Automation, Hong Kong, China, May. 2014, pp. 6278-6284. 